\title{
EVEN LENGTH PALINDROMES
}

\author{
THINH D. NGUYEN
}

\section{Problem statement}

Definition 1. Even Palindrome problem:

Input: Given a list of strings $\left[v_{1}, v_{2}, \ldots, v_{n}\right]$ where $\Sigma\left|v_{i}\right|$ is even number.

Question: Is there a permutation $\sigma$ such that $w=v_{\sigma(1)} v_{\sigma(2)} \ldots v_{\sigma(n)} \in\left\{u u^{R} \in\right.$ $\left.\Sigma^{*}\right\}$.

In other word, our problem is determining if a given set of strings can be ordered to form a palindrome (of even length as defined above). Morover, we can restrict the cardinality of the alphabet $|\Sigma|=2$ ?

We reduce 3-PARTITION problem to ours. 3-PARTITION is shown to be strongly hard by [1]. We claim that even with a binary alphabet, our problem is a very hard one.

Claim 2. We have that 3-Partition $\leq_{p}$ Even Palindrome

\section{Reducing 3-Partition to Even Palindrome}

The 3-partition problem is to decide whether a given multiset $S=\left\{a_{1}, a_{2}, \ldots, a_{n}\right\}$ of $n=3 m$ positive integers, can $S$ be partitioned into $m$ triplets $S_{1}, S_{2}, \ldots, S_{m}$ such that the sum of the numbers in each subset is equal?

Let $p=\left(\sum_{i} a_{i}\right) / m$

Note that the problem remains strongly NP-complete even if $p / 4<a_{i}<p / 2$ (which forces each $S_{i}$ to contain at exactly three elements).

We can build an equivalent Even PALINDrome instance in the following way:

We add the long string:

$$
w=10^{p} 1110^{p} 111110^{p} \ldots 0^{p} 1^{2 m+1}
$$

Then we add $n$ more strings:

$$
v_{i}=0^{a_{i}}, i=1 \ldots n
$$

and $1+3+5+(2 m+1)=\sum_{j=1}^{m+1}(2 j-1)$ strings of length 1 :

$$
r_{k}=1, k=1 \ldots \sum_{j=1}^{m+1}(2 j-1)
$$

Note that $|w|=\sum_{i=1}^{n}\left|v_{i}\right|+\sum_{k}\left|r_{k}\right|$

Key words and phrases. alphabet, palindrome, sequence, string.

Perebor. 
Proof. By construction the $w$ contains substrings of odd increasing length $(1,111,11111, \ldots)$, so it cannot be "placed" in the middle of $u u^{R}$; but it must be placed entirely in $u$ or entirely in $u^{R}$ (without loss of generality suppose that it is placed in $u$.

Now the others $v_{i}$ must be arranged in the opposite part $u^{R}$ and in such a way that every $p$ zeroes (that can be "totalized" using the $v_{i}$ ) an odd number of the $r_{k}$ can be placed (to reflect the corresponding $1^{2 j+1}$ substring of $w$ ). Furthermore all elements must be placed, so exactly $3 v_{i}$ must be picked to reflect each of the $0^{p}$ substring of $w$. So if an EVEN PALINDROME can be found it induces a valid 3 -partition of the $a_{i}$ of the original problem.

The opposite direction (a 3-partition exists $\Rightarrow$ there is an Even PALINDrome is immediate by construction).

Example:

3-Partition instance: $S=\{1,1,1,2,2,3\}$, strongly NP-complete; so we can also represent the numbers in unary: $S^{\prime}=\left\{1_{1}, 1_{1}, 1_{1}, 11_{1}, 11_{1}, 111_{1}\right\}$

Even PALINDROME reduction:

$w=1000001110000011111$

$v_{i}=\{0,0,0,00,00,000\}$

$r_{k}=\{1, \quad 1,1,1, \quad 1,1,1,1,1\}$

Solution of Even PALINDRome:

$$
\left.u u^{R}=10000011100000111111111100000111000001\right\}
$$

which corresponds to the 3 -partition:

$S_{1}=\{1,2,2\}, S_{2}=\{3,1,1\}$

\section{Conclusion}

Garey and Johnson [1] shapes their theory based on previous primal works of Cook, Levin and Karp. Johnson [2] moves on with the guide to this theory. As long as we study a mathematical conjecture, we should encourage ourselves of having enough labouring hours on popular maths books like these. Then, reading some articles on theory of computing like [3] is a good practice. Only after that, could we think of the ultimate final for all mathematics sciences.

\section{REFERENCES}

1. Michael R. Garey, David S. Johnson, Computers and Intractability: A Guide to the Theory of NP-Completeness

2. David S. Johnson, The NP-Completeness Column: An Ongoing Guide

3. Phan Dinh Dieu, Le Cong Thanh, Le Tuan Hoa, Average Polyno-mial Time Complexity of Some NP-Complete Problems, Theor. Comput. Sci. 46(3): pp.219-237 (1986)

Current address: Department of Mathematics, Moscow State University

Email address: kosmofarmer@yandex.com 